MPP-2007-15

\title{
Sensitivity of low energy neutrino experiments to physics beyond the Standard Model
}

\author{
J. Barrancd* and O. G. Mirandat \\ Departamento de Fúsica, Centro de Investigación y de Estudios Avanzados del IPN, \\ Apartado Postal 14-740 07000 México, D F, México \\ T. I. Rashba \\ Max-Planck-Institut für Physik (Werner-Heisenberg-Institut), \\ Föhringer Ring 6, 80805 München, Germany and \\ Institute of Terrestrial Magnetism, Ionosphere and Radio \\ Wave Propagation of the Russian Academy of Sciences, \\ 142190, Troitsk, Moscow region, Russia
}

(Dated: September 18, 2018)

\begin{abstract}
We study the sensitivity of future low energy neutrino experiments to extra neutral gauge bosons, leptoquarks and $R$-parity breaking interactions. We focus on future proposals to measure coherent neutrino-nuclei scattering and neutrino-electron elastic scattering. We introduce a new comparative analysis between these experiments and show that in different types of new physics it is possible to obtain competitive bounds to those of present and future collider experiments. For the cases of leptoquarks and $R$-parity breaking interactions we found that the expected sensitivity for most of the future low energy experimental setups is better than the current constraints.
\end{abstract}

\footnotetext{
*Electronic address: jbarranc@fis.cinvestav.mx

${ }^{\dagger}$ Electronic address: Omar.Miranda@fis.cinvestav.mx

‡Electronic address: timur@mppmu.mpg.de
} 


\section{INTRODUCTION}

The Standard Model (SM) is one of the most successful models in physics and it is in very good agreement with almost every measurement in high energy physics[1]. Despite this fact, there are many motivations to believe that the SM is not the last step in the description of the physics of elementary particles.

There are many theoretical motivations to believe that there is physics beyond the standard model, and recently the neutrino oscillation experiments have also given an experimental input on these thoughts. Among the most popular extensions of the SM we find grand unified theories (GUT), supersymmetry (SUSY), and extra dimensions. None of these theories have been observed in the laboratory, but there are extensive searches for signatures of them in collider physics. The main aim of this paper is to analyze the potential of low energy neutrino experiments either to confirm the presence of new physics if it would be discovered by the Large Hadron Collider (LHC), or put stronger or complementary constraints on their parameters.

We center our attention in signatures that could appear in two different reactions: coherent neutrino-nuclei scattering and neutrino-electron elastic scattering. As concrete examples of coherent neutrino-nuclei scattering we will consider the TEXONO proposal [2], a stopped pion source (SPS) with a noble gas detector [3] and the recently discussed proposal of low energy beta beams [4]. For the neutrino electron scattering case, we concentrate in the Double Chooz proposal [5].

For some of these experimental proposals there have already been discussions about their perspectives for constraining non-standard neutrino interactions [3, 7] or a non-zero neutrino magnetic moment [3, 8, 9, 10]. In this work we introduce a new comparative analysis between different low energy experiments, focusing on three different types of new physics phenomenology, namely extra neutral gauge bosons, leptoquarks and $R$-parity breaking Supersymmetry. As far as we know, this is the first time that the sensitivity of low energy neutrino proposals to leptoquarks is studied. On the other hand, extra neutral gauge bosons sensitivity had been studied only for the TEXONO and neutrino electron scattering proposals [7, 9, 11]. For the case of $R$-parity breaking Supersymmetry the existing studies have tested either long-baseline neutrino experiments [12] that introduce an extra dependence on $\theta_{13}$, or new physics effects in the source due to charged currents [13], while here we will focus 
on neutral currents effects, visible in the detector, specifically in a short baseline detector based on coherent neutrino nuclei scattering. Moreover, the study of different future proposals at one time gives to the reader an extra usefulness of telling which future experiments will give better chances in the different types of new physics under study. We will see that, despite the fact that we are dealing with very low energy experiments there are good chances to obtain a very good sensitivity to these types of new physics and either to compete or to give complementary constraints to those that could be obtained from collider experiments.

The structure of the article is the following: In section II we describe the experimental proposals that we study. In section III we introduce the different types of new physics under consideration and the expected sensitivity in the different experimental setups. Finally in section IV we present our conclusions.

\section{EXPERIMENTAL PROPOSALS}

Before introducing the phenomenology to study new physics signatures we would like to discuss the low energy neutrino experimental proposals. In particular, we will discuss the case of future experiments aiming to measure the coherent neutrino scattering off nuclei as well as the case of low energy neutrino-electron-scattering experiments. For the first reaction we study three different recent proposals while for neutrino-electron scattering we concentrate in the Double Chooz case.

\section{A. TEXONO}

TEXONO collaboration has recently started a research program towards the measurement of neutrino-nuclei coherent scattering by using reactor neutrinos and an "ultralow-energy" germanium detector (ULEGe) [2].

The proposed detector would consist of $1 \mathrm{~kg}$ of an ultralow-energy germanium detector with a threshold as low as $100 \mathrm{eV}$ and a background level below $1 \mathrm{keV}$ in the range of 1 count-per-day that implies a signal to noise ratio bigger than 22. Although an estimate for the systematic uncertainties is not available, we can consider that they will be dominated by the reactor power, its fuel composition, and the anti-neutrino spectrum. We assume that these uncertainties will give an approximate error of $2 \%$ [14]. 
Besides the $100 \mathrm{eV}$ threshold, we will also consider the more conservative case of a $400 \mathrm{eV}$ threshold. The typical time scale of data taking is assumed to be from one to several years.

The electron anti-neutrino flux is coming from the Kuo-Sheng Nuclear Power Station. The detector will be located at a distance of $28 \mathrm{~m}$ from the reactor core. In our computation we will assume a typical reactor neutrino flux of $10^{13} \mathrm{~s}^{-1} \mathrm{~cm}^{-2}$. There are several parameterizations that consider in detail the neutrino spectrum coming from a reactor [14, 15, 16]. In this work we will use the most recent parameterization [14] for the neutrino spectrum. Since the proposed experiments are not running yet, we will assume that the relative contribution of the fissile isotopes $\left({ }^{235} \mathrm{U},{ }^{239} \mathrm{Pu},{ }^{238} \mathrm{U},{ }^{241} \mathrm{Pu}\right)$ is given by the typical average values of the reactor operating period [17] which is given by $0.58: 0.30: 0.07: 0.05$. We have checked numerically that the results does not change significantly with other ratios. For energies below $2 \mathrm{MeV}$ there are only theoretical calculations for the antineutrino spectrum that we take from Ref. [17].

Since we are not able to account for the detector efficiency and resolution, we will estimate the total number of expected events in a detector as

$$
N_{\text {events }}^{T E X O N O}=t \phi_{0} \frac{M_{\text {detector }}}{M} \int_{0}^{E_{\max }} d E_{\nu} \int_{T_{t h}}^{T_{\max }\left(E_{\nu}\right)} d T \lambda\left(E_{\nu}\right) \frac{d \sigma}{d T}\left(E_{\nu}, T\right),
$$

with $t$ the data taking time period, $\phi_{0}$ the total neutrino flux, $M_{\text {detector the total mass of }}$ the detector, $\lambda\left(E_{\nu}\right)$ the normalized neutrino spectrum, $E_{\max }$ the maximum neutrino energy, $T_{t h}$ the detector energy threshold. The maximal recoil energy is $T_{\max }\left(E_{\nu}\right)=2 E_{\nu}^{2} /(M+$ $\left.2 E_{\nu}\right)$. The same expression relates the minimum required incoming neutrino energy with the detector threshold $T_{t h}$. For instance, for the detector's threshold $400 \mathrm{eV}$ and ${ }^{76} \mathrm{Ge}$ nucleus, the minimum required incoming neutrino energy is about $3.8 \mathrm{MeV}$ which is well satisfied for reactor neutrinos.

\section{B. Stopped pion neutrino source}

A different proposal for detecting the coherent neutrino-nucleus scattering considers the use of another source of neutrinos, a SPS, such as the Spallation Neutron Source at Oak Ridge National Laboratory. Recently, this type of source was proposed to measure coherent neutrino scattering off nuclei as well as non-standard neutrino properties [3].

The total beam flux consists of the following well-known neutrino fluxes: 
- the monoenergetic $29.9 \mathrm{MeV} \nu_{\mu}$ 's produced from pion decay at rest, $\pi^{+} \rightarrow \mu^{+} \nu_{\mu}$;

- $\bar{\nu}_{\mu}$ and $\nu_{e}$ coming from muon decay, $\mu^{+} \rightarrow e^{+} \nu_{e} \bar{\nu}_{\mu}$, with a time delay about $2.2 \mu \mathrm{s}$, muon decay time scale.

The neutrino spectra are well known. Here we will consider only the total delayed flux $\left(\nu_{e}+\overline{\nu_{\mu}}\right)$ as was done in Ref. [3]. We assume a total flux of $\sim 10^{7} \nu \mathrm{s}^{-1} \mathrm{~cm}^{-2}$. Among different possible detector materials such as Ar, Ge or Xe, we will concentrate on the noble gas detector, ${ }^{20} \mathrm{Ne}$, of typical mass about $100 \mathrm{~kg}$ with a data taking time scale from one to several years and a threshold of $10 \mathrm{keV}$.

\section{Low energy beta beams}

The usage of accelerated radioactive nuclei to produce a well known flux of neutrinos - beta beam - was proposed in [18]. It was shown soon afterwards that low energy beta beams open new possibilities to study neutrino properties [19] and, recently, a neutrinonuclei coherent scattering experiment using neutrinos from low energy beta beams was

discussed [4]. On the other hand, tests for $R$ parity violating Supersymmetry have been discussed both by the direct detection of $\tau$ leptons in a nearby detector [13] as well as in long baseline beta-beam experiments [12].

In particular we base our analysis on the beta-beam experiment discussed in [4, 20]. We consider a storage ring of total length $L=1885 \mathrm{~m}$ with a straight sections of length $D=678 \mathrm{~m}$. In the stationary regime the mean number of ions in the storage ring is $\gamma \tau g$, where $\tau=t_{1 / 2} / \ln 2$ is the lifetime of the parent nuclei, $g=2.7 \times 10^{12}$ is the number of injected ions per second and $\gamma=1 / \sqrt{1-\beta^{2}}$ is the time delay factor with $\beta$ the ion velocity in the laboratory frame. As previous authors, we will consider a cylindrical detector of radius $R=52 \mathrm{~cm}$ and depth $h=40 \mathrm{~cm}$, aligned with one of the storage ring's straight sections, and located at a distance $d=10 \mathrm{~m}$ from it. Integration over the decay path and over the volume of the detector gives the total number of events per unit time

$$
N_{\text {events }}^{\beta-\text { beam }}=\operatorname{tg} \tau n h \times \int_{0}^{\infty} d E_{\nu} \Phi_{\text {tot }}\left(E_{\nu}\right) \sigma\left(E_{\nu}\right),
$$

where $t=1$ year is the data taking time, $n$ is the number of target nuclei per unit volume, $\sigma\left(E_{\nu}\right)$ is the relevant neutrino-nucleus cross-section. For definiteness we consider the case 
of a ton of Xe as a target and a factor $\gamma=14$ for ${ }^{6} \mathrm{He}$ ions as described in Ref. [4]. As for the threshold energy, we consider both the realistic threshold of $15 \mathrm{keV}$ where background events are negligible as well as the very optimistic $5 \mathrm{keV}$ threshold that, according to the same reference, will give a bigger number of events if background could be subtracted, though at present there is no technology capable of dealing with such a background. The total neutrino flux through detector is given by

$$
\Phi_{t o t}\left(E_{\nu}\right)=\int_{0}^{D} \frac{d \ell}{L} \int_{0}^{h} \frac{d z}{h} \int_{0}^{\bar{\theta}(\ell, z)} \frac{\sin \theta d \theta}{2} \Phi_{l a b}\left(E_{\nu}, \theta\right)
$$

where

$$
\tan \bar{\theta}(\ell, z)=\frac{R}{d+\ell+z}
$$

The boosted flux in the laboratory frame is

$$
\Phi_{l a b}\left(E_{\nu}, \theta\right)=\frac{\Phi_{c m}\left(E_{\nu} \gamma[1-\beta \cos \theta]\right)}{\gamma[1-\beta \cos \theta]},
$$

where $E_{\nu}$ and $\Omega \equiv(\theta, \varphi)$ denote the energy and solid angle of the emitted (anti-)neutrino in the laboratory $(l a b)$ frame and $\theta$ denotes the angle of emission with respect to the beam axis.

The neutrino flux in the rest frame, $\Phi_{c m}\left(E_{\nu}^{\prime}\right)$, is given by the well-known formula [21]:

$$
\Phi_{c m}\left(E_{\nu}^{\prime}\right)=\frac{\ln 2}{m_{e}^{5} f t_{1 / 2}}\left(E_{\nu}^{\prime}\right)^{2} E_{e} \sqrt{E_{e}^{2}-m_{e}^{2}} F\left( \pm Z, E_{e}\right) \Theta\left(E_{e}-m_{e}\right)
$$

where $m_{e}$ the electron mass and $f t_{1 / 2}$ the $f t$ value. The energy of emitted lepton (electron or positron) is $E_{e}=Q-E_{\nu}^{\prime}$, where $Q$ is the $Q$ value of the reaction, and the Fermi function $F\left( \pm Z, E_{e}\right)$ accounts for the Coulomb modification of the spectrum [22].

\section{Reactor experiments}

A different type of experiment that we will also consider in this article is based on low energy neutrino-electron scattering. This process has already been considered as a possible place to search for an extra gauge boson [11, 23]. The case of a reactor source to constrain new physics has recently been discussed both for present [24] and future proposals [9]. In this work we will concentrate on the perspectives for the Double Chooz experiment [5]. As in [9], we assume that the Double Chooz will collect $10^{4}$ neutrino-electron-scattering events considering a $3 \mathrm{GW}$ reactor and a 26.5 ton detector with an electron visible energy window 


\begin{tabular}{|c|c|c|c|}
\hline Experiment & $M_{0}$ & Expected events/yr & systematic error estimate \\
\hline Texono, $E_{t h}=400 \mathrm{eV}$ & $1 \mathrm{~kg}, \mathrm{Ge}$ & 3790 & $2 \%$ \\
Texono, $E_{t h}=100 \mathrm{eV}$ & $1 \mathrm{~kg}, \mathrm{Ge}$ & 25196 & $2 \%$ \\
Beta beam, $E_{t h}=15 \mathrm{keV}$ & $1 \mathrm{ton}, \mathrm{Xe}$ & 1390 & $2 \%$ \\
Beta beam, $E_{t h}=5 \mathrm{keV}$ & $1 \mathrm{ton}, \mathrm{Xe}$ & 5309 & $2 \%$ \\
Stopped pion, $E_{t h}=10 \mathrm{keV}$ & $100 \mathrm{~kg}, \mathrm{Ne}$ & 627 & $5 \%$ \\
Double Chooz & 26.5 tons, scintill. & 10000 & $1 \%$ \\
\hline
\end{tabular}

TABLE I: Expected events for different experimental setups

$3 \mathrm{MeV}<T<5 \mathrm{MeV}$. As in the case of the TEXONO proposal, we will use the most recent parameterization [14] for the neutrino spectrum and the same fuel composition.

\section{E. Discussion on experiments}

We summarize the main characteristics of the detectors in Table I. One can notice that in some cases it could be possible to run the experiment for a period longer than one year, or to upgrade the detector mass, obtaining a smaller statistical error without being dominated by systematic uncertainties. This is the case, for example, for a beta beam with a $15 \mathrm{keV}$ threshold. On the other hand the Stopped pion source seems to be suitable only for a one year of data taking. Finally we also consider the very optimistic cases in which experimentalists can reduce the uncertainties in a low threshold regime (like a beta beam with a $5 \mathrm{keV}$ threshold). In this case we assume that the systematic uncertainties remain the same.

In the next sections we will take into account all these experimental setups. We will also show results for possible upgrades to these experiments, i.e., we will consider that the experimental setup can be running for a longer time (or that an upgrade in mass is possible). Among the difficulties for the upgrade we must take special care of the systematic error expectations. In order to take a reasonable compromise with future experimental capabilities, we will consider the systematic errors quoted in Table I. Since we are dealing with experiments that are not running yet, we believe that this approach will be helpful to take notice of what would be the expected limits for each experiment. 


\section{MODELS AND SENSITIVITY}

Once the experimental setups have been discussed, we turn our attention to different types of new physics that could be constrained in these future proposals. We will consider three different scenarios that will be discussed in detail in the following subsections.

\section{A. $Z^{\prime}$ models}

In this section we introduce the description of the extra gauge bosons to be considered. New massive gauge bosons are a common feature of physics beyond the Standard Model. Heavy neutral vector bosons $Z^{\prime}$ are predicted in string inspired extensions of the SM, in left-right symmetric models, in models with dynamical symmetry breaking, in "little Higgs" models and in certain classes of theories with extra dimensions. In many of these models it is expected that $Z^{\prime}$ mass can be around $\mathrm{TeV}$ scale.

The present experimental lower limits to the neutral gauge boson mass come from the Tevatron and LEP experiments [1]. Forthcoming measurements at LHC will provide sensitivity to the $Z^{\prime}$ mass up to $5 \mathrm{TeV}[25,26]$.

The new $Z^{\prime}$ boson affects the neutral current couplings of the SM, and its contribution at low energies can be tested from atomic parity violation and by electron-nucleon scattering experiments (see refs in [1] ). Since low energy experiments are not sensitive to the mixing angle between the SM gauge boson and the extra gauge boson, and this angle is very well constrained [1], we will neglect it.

We consider first the particular case of an additional neutral gauge boson $Z^{\prime}$ that arises from a primordial $E_{6}$ gauge symmetry [27]. These extension usually involve an extra $U(1)$ hypercharge symmetry at low energies that may be given as the mixture of those associated with the symmetries $U(1)_{\chi}$ and $U(1)_{\psi}$. We show the quantum numbers for the SM particles in Table ஹ,

The corresponding hyper-charge is then specified by

$$
Y_{\beta}=Y_{\chi} \cos \beta+Y_{\psi} \sin \beta
$$

while the charge operator is given as $Q=T^{3}+Y$. Any value of $\beta$ is allowed, giving us a continuum spectrum of possible models of the weak interaction. At tree level it is possible to write an expression for the effective 4-fermion Lagrangian describing low-energy neutral 
TABLE II: Quantum numbers for the light particles in the $\mathbf{2 7}$ of $E_{6}$.

\begin{tabular}{|c|c|c|c|}
\hline \multicolumn{2}{|r|}{$T_{3}$} & \multicolumn{2}{|c|}{$\sqrt{40} Y_{\chi} \sqrt{24} Y_{\psi}$} \\
\hline$Q$ & $\left.\begin{array}{c}1 / 2 \\
-1 / 2\end{array}\right)$ & -1 & 1 \\
\hline$u^{c}$ & 0 & -1 & 1 \\
\hline$e^{c}$ & 0 & -1 & 1 \\
\hline$d^{c}$ & 0 & 3 & 1 \\
\hline$l$ & $\left.\begin{array}{c}1 / 2 \\
-1 / 2\end{array}\right)$ & 3 & 1 \\
\hline
\end{tabular}

current phenomena. We neglect nonstandard radiative corrections because its contribution is of order $(\alpha / \pi)\left(M_{Z}^{2} / M_{Z^{\prime}}^{2}\right)[28]$. Another class of $Z^{\prime}$ models is coming from left-right symmetric models that have the premise that the fundamental weak interaction Lagrangian is invariant under parity symmetry at energies about $100 \mathrm{GeV}$. The gauge group of this type of models is given by $S U(2))_{L} \otimes S U(2)_{R} \otimes U(1)_{B-L}$, which gives an additional neutral gauge boson plus a charge gauge boson [29, 30]. We will concentrate in this work on the neutral currents.

In the following subsections we will introduce the modifications to the coupling constants, and therefore to the cross section, due to this type of new physics. With this information we will study the different experimental proposals and their sensitivity to both $E_{6}$ and left-right symmetric neutral gauge bosons.

\section{Coherent neutrino-nuclei scattering coupling constants}

Before introducing this description it is useful to recall the general description of the nonstandard neutrino-quark and neutrino-electron interactions and then we will specify the interactions for commonly used $Z^{\prime}$ models.

Generically the neutrino-quark interaction at low energies (energies $\ll M_{Z}$ ) can be described at the 4-fermion approximation by the effective Lagrangian

$$
\mathcal{L}_{\nu \text { Hadron }}^{N C}=-\frac{G_{F}}{\sqrt{2}} \sum_{q=u, d}\left[\bar{\nu}_{e} \gamma^{\mu}\left(1-\gamma^{5}\right) \nu_{e}\right]\left(f^{q L}\left[\bar{q} \gamma_{\mu}\left(1-\gamma^{5}\right) q\right]+f^{q R}\left[\bar{q} \gamma_{\mu}\left(1+\gamma^{5}\right) q\right]\right)
$$

where

$$
f^{u L}=\rho_{\nu N}^{N C}\left(\frac{1}{2}-\frac{2}{3} \hat{\kappa}_{\nu N} \hat{s}_{Z}^{2}\right)+\lambda^{u L}+\varepsilon^{u L},
$$




$$
\begin{aligned}
f^{d L} & =\rho_{\nu N}^{N C}\left(-\frac{1}{2}+\frac{1}{3} \hat{\kappa}_{\nu N} \hat{s}_{Z}^{2}\right)+\lambda^{d L}+\varepsilon^{d L}, \\
f^{u R} & =\rho_{\nu N}^{N C}\left(-\frac{2}{3} \hat{\kappa}_{\nu N} \hat{s}_{Z}^{2}\right)+\lambda^{u R}+\varepsilon^{u R} \\
f^{d R} & =\rho_{\nu N}^{N C}\left(\frac{1}{3} \hat{\kappa}_{\nu N} \hat{s}_{Z}^{2}\right)+\lambda^{d R}+\varepsilon^{d R}
\end{aligned}
$$

Here $\hat{s}_{Z}^{2}=\sin ^{2} \theta_{W}=0.23120$ - the Weinberg weak mixing angle taken in the $\overline{\mathrm{M} S}$ model. The radiative corrections [1] $\rho_{\nu N}^{N C}=1.0081, \hat{\kappa}_{\nu N}=0.9978, \lambda^{u L}=-0.0031, \lambda^{d L}=-0.0025$, and $\lambda^{d R}=2 \lambda^{u R}=7.5 \times 10^{-5}$ are included into our analysis. In general, the parameters $\varepsilon^{q P}(q=u, d$ and $P=L, R)$ describe a generic nonstandard neutrino interaction. For the specific case of $E_{6}$ string inspired models this is translated into

$$
\begin{aligned}
& \varepsilon^{u L}=-4 \gamma \sin ^{2} \theta_{W} \rho_{\nu N}^{N C}\left(\frac{c_{\beta}}{\sqrt{24}}-\frac{s_{\beta}}{3} \sqrt{\frac{5}{8}}\right)\left(\frac{3 c_{\beta}}{2 \sqrt{24}}+\frac{s_{\beta}}{6} \sqrt{\frac{5}{8}}\right) \\
& \varepsilon^{d R}=-8 \gamma \sin ^{2} \theta_{W} \rho_{\nu N}^{N C}\left(\frac{3 c_{\beta}}{2 \sqrt{24}}+\frac{s_{\beta}}{6} \sqrt{\frac{5}{8}}\right)^{2}, \\
& \varepsilon^{d L}=\varepsilon^{u L}=-\varepsilon^{u R},
\end{aligned}
$$

where $c_{\beta}=\cos \beta, s_{\beta}=\sin \beta$ and $\gamma=\left(M_{Z} / M_{Z^{\prime}}\right)^{2}$. Three main models have been extensively studied, namely: the $\chi$ model $(\cos \beta=1)$, the $\psi$ model $(\cos \beta=0)$ and the $\eta$ model $(\cos \beta=\sqrt{3 / 8})$. In previous articles [23] it has been stressed that low energy neutrino experiments are more sensitive to the $\chi$ model than to other $E_{6}$ models. However, for comparison with the expected sensitivity to $Z^{\prime}$ mass in different models at LHC we will consider a continuum spectrum of possible models over parameter $\beta$.

From the Lagrangian in Eq. (8) we can obtain the coherent neutrino-nucleus differential cross section which is given by

$$
\frac{d \sigma}{d T}=\frac{G_{F}^{2} M}{2 \pi}\left\{\left(G_{V}+G_{A}\right)^{2}+\left(G_{V}-G_{A}\right)^{2}\left(1-\frac{T}{E_{\nu}}\right)^{2}-\left(G_{V}^{2}-G_{A}^{2}\right) \frac{M T}{E_{\nu}^{2}}\right\}
$$

where $M$ is the mass of the nucleus, $T$ is the recoil nucleus energy, which varies from 0 to $T_{\max }=2 E_{\nu}^{2} /\left(M+2 E_{\nu}\right), E_{\nu}$ is the incident neutrino energy and

$$
\begin{aligned}
& G_{V}=\left[\left(g_{V}^{p}+2 \varepsilon_{e e}^{u V}+\varepsilon_{e e}^{d V}\right) Z+\left(g_{V}^{n}+\varepsilon_{e e}^{u V}+2 \varepsilon_{e e}^{d V}\right) N\right] F_{n u c l}^{V}\left(Q^{2}\right), \\
& G_{A}=\left[\left(g_{A}^{p}+2 \varepsilon_{e e}^{u A}+\varepsilon_{e e}^{d A}\right)\left(Z_{+}-Z_{-}\right)+\left(g_{A}^{n}+\varepsilon_{e e}^{u A}+2 \varepsilon_{e e}^{d A}\right)\left(N_{+}-N_{-}\right)\right] F_{n u c l}^{A}\left(Q^{2}\right) .
\end{aligned}
$$

$Z$ and $N$ represent the number of protons and neutrons in the nucleus, while $Z_{ \pm}\left(N_{ \pm}\right)$stands for the number of protons (neutrons) with spin-up and spin-down respectively. From Eq. (13) it is possible to see that the axial couplings will vanish for even-even nuclei considered below. 
The vector and axial nuclear form factors, $F_{n u c l}^{V}\left(Q^{2}\right)$ and $F_{n u c l}^{A}\left(Q^{2}\right)$, are usually assumed to be equal and of order of unity in the limit of small energies, $Q^{2} \ll M^{2}$. In our computations, for the sake of completeness we take into account the vector form factor given in Ref. [31]. We have also made our computations taking into account previous calculations of this form factor [32, 33, 34], and we found that there is no difference in our results for both of them, which give us confidence to consider that this theoretical estimation will not have an impact on the systematic errors. The SM neutral current vector couplings of neutrinos with protons, $g_{V}^{p}$, and with neutrons, $g_{V}^{n}$, are defined as

$$
\begin{aligned}
& g_{V}^{p}=\rho_{\nu N}^{N C}\left(\frac{1}{2}-2 \hat{\kappa}_{\nu N} \hat{s}_{Z}^{2}\right)+2 \lambda^{u L}+2 \lambda^{u R}+\lambda^{d L}+\lambda^{d R}, \\
& g_{V}^{n}=-\frac{1}{2} \rho_{\nu N}^{N C}+\lambda^{u L}+\lambda^{u R}+2 \lambda^{d L}+2 \lambda^{d R}
\end{aligned}
$$

Besides string inspired models, we also consider left-right symmetric models. In this case the coupling constants in Eq. (9) can be expressed as [35]

$$
\begin{aligned}
f^{u L} & =\rho_{\nu N}^{N C} A\left(\frac{1}{2}-\frac{2}{3} \hat{\kappa}_{\nu N} \hat{s}_{Z}^{2}\right)-B \frac{2}{3} \hat{s}_{Z}^{2}+\lambda^{u L} \\
f^{d L} & =\rho_{\nu N}^{N C} A\left(-\frac{1}{2}+\frac{1}{3} \hat{\kappa}_{\nu N} \hat{s}_{Z}^{2}\right)+B \frac{1}{3} \hat{s}_{Z}^{2}+\lambda^{d L} \\
f^{u R} & =\rho_{\nu N}^{N C} A\left(-\frac{2}{3} \hat{\kappa}_{\nu N} \hat{s}_{Z}^{2}\right)+B\left(\frac{1}{2}-\frac{2}{3} \hat{s}_{Z}^{2}\right)+\lambda^{u R} \\
f^{d R} & =\rho_{\nu N}^{N C} A\left(\frac{1}{3} \hat{\kappa}_{\nu N} \hat{s}_{Z}^{2}\right)+B\left(-\frac{1}{2}+\frac{1}{3} \hat{s}_{Z}^{2}\right)+\lambda^{d R}
\end{aligned}
$$

where

$$
\begin{aligned}
& A=1+\frac{\hat{s}_{Z}^{4}}{1-2 \hat{s}_{Z}^{2}} \gamma \\
& B=\frac{\hat{s}_{Z}^{2}\left(1-\hat{s}_{Z}^{2}\right)}{1-2 \hat{s}_{Z}^{2}}
\end{aligned}
$$

\section{Neutrino-electron scattering coupling constants}

For the case of neutrino-electron scattering the total Lagrangian has the form

$$
\mathcal{L}_{\nu e}^{N C}=-\frac{G_{F}}{\sqrt{2}} \sum_{\alpha, \beta=e, \mu, \tau}\left[\bar{\nu}_{\alpha} \gamma^{\mu}\left(1-\gamma^{5}\right) \nu_{\beta}\right]\left(f^{e L}\left[\bar{e} \gamma_{\mu}\left(1-\gamma^{5}\right) e\right]+f^{e R}\left[\bar{e} \gamma_{\mu}\left(1+\gamma^{5}\right) e\right]\right)
$$


with $f^{e L, R}=g_{L, R} \pm \varepsilon^{L, R}$, and

$$
\begin{aligned}
\varepsilon^{L} & =2 \gamma \sin ^{2} \theta_{W} \rho_{\nu e}^{N C}\left(\frac{3 c_{\beta}}{2 \sqrt{6}}+\frac{s_{\beta}}{3} \sqrt{\frac{5}{8}}\right)^{2} \\
\varepsilon^{R} & =2 \gamma \sin ^{2} \theta_{W} \rho_{\nu e}^{N C}\left(\frac{c_{\beta}}{2 \sqrt{6}}-\frac{s_{\beta}}{3} \sqrt{\frac{5}{8}}\right)\left(\frac{3 c_{\beta}}{\sqrt{24}}+\frac{s_{\beta}}{3} \sqrt{\frac{5}{8}}\right) .
\end{aligned}
$$

As in the previous subsection, here $\gamma=\left(M_{Z} / M_{Z^{\prime}}\right)^{2}$. With this Lagrangian, the neutrinoelectron scattering will keep the same form

$$
\frac{d \sigma}{d T}=\frac{2 G_{F} m_{e}}{\pi}\left[g_{L}^{2}+g_{R}^{2}\left(1-\frac{T}{E_{\nu}}\right)^{2}-g_{L} g_{R} \frac{m_{e} T}{E_{\nu}^{2}}\right]
$$

with the only difference that now the coupling constants $g_{L, R}$ will be defined as

$$
\begin{aligned}
g_{L} & =\frac{1}{2}+\sin ^{2} \theta_{W}+\varepsilon^{L} \\
g_{R} & =\sin ^{2} \theta_{W}+\varepsilon^{R} .
\end{aligned}
$$

For the left-right symmetric case, we can express the coupling constants as

$$
\begin{aligned}
& g_{L}^{L R}=A g_{L}+B g_{R} \\
& g_{R}^{L R}=A g_{R}+B g_{L}
\end{aligned}
$$

where $A$ and $B$ were defined in Eq. (16).

\section{Future sensitivity}

In order to compute the expected $Z^{\prime}$ mass limit that these experiments could get, we consider that the future experiment will measure exactly the Standard Model prediction, and we add the systematic error in quadratures to the statistical one. With these hypothesis we can compute the $95 \%$ C.L. bound reachable at these future experiments after one year of data taking.

We make this computation for the string inspired models for all possible values of $\cos \beta$ considering the detector characteristics explained in the previous section. The results are shown in Fig. 1, where we also show, for comparison, the current constraints at 95\% C.L. [1]. Note that the expectations for the Double Chooz experiment are in a qualitative agreement with similar analysis done before the MUNU experiment in Ref. [11]. For the left-right symmetric case the expected sensitivity is shown in Table III. 


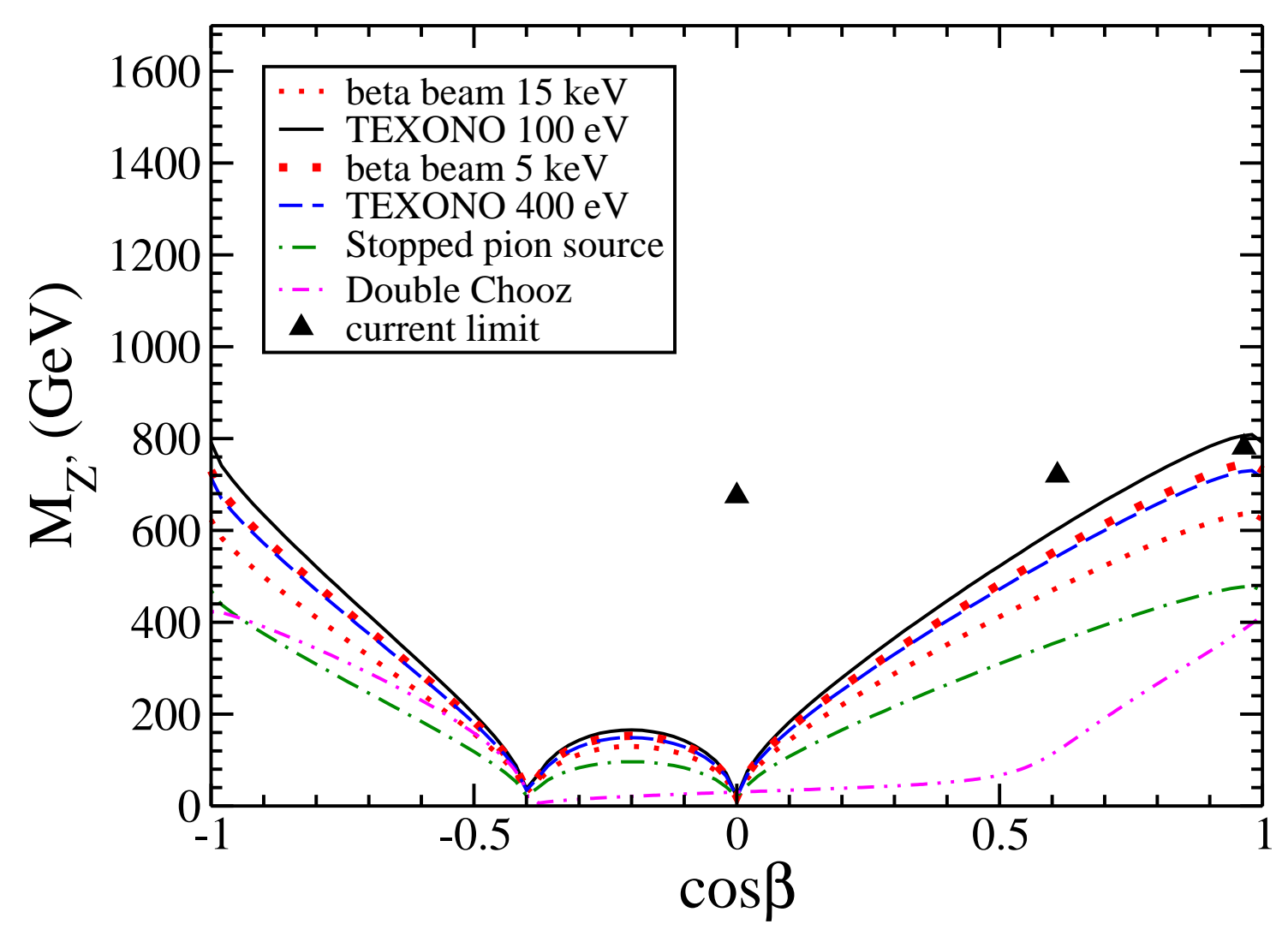

FIG. 1: Sensitivity, at 95\% CL, to different extra neutral gauge boson coming from $E_{6}$ models. We consider the case of the TEXONO proposal for an energy threshold of $100 \mathrm{eV}$ (solid line) and $400 \mathrm{eV}$ (dashed line); the case of a future stopped-pion source (dash-dotted line) and a beta-beam source with energy threshold of $15 \mathrm{keV}$ (bold dotted line) and $5 \mathrm{keV}$ (dotted line). Finally, the Double Chooz sensitivity is also shown (dashed double dotted). The current limits (triangles) are also shown for comparison.

From Fig. 1 it is possible to see different phenomenological aspects. First, the $\chi$ model $(\cos \beta=1)$ is the most sensitive for low energy neutrino experiments. Second, for the coherent neutrino-nucleus scattering case, the $\psi$ model $(\cos \beta=0)$ is in the opposite situation. This behavior is clear from Eq. (10), that for this specific value the corrections to the Standard Model Lagrangian cancel. A similar property arises both in the case of coherent neutrino-nucleus scattering as well as in anti-neutrino-electron scattering for $\cos \beta=-\sqrt{5 / 32}$. These features of different specific models seems to discourage the search for this type of new physics in low energy neutrino experiments, since only a few models can give a significant signature. However, in the case of a positive signature in LHC we can 


\begin{tabular}{|c|c|c|c|c|c|c|c|}
\hline Experiment & $\begin{array}{c}\text { Texono } \\
100 \mathrm{eV}\end{array}$ & $\begin{array}{c}\text { Beta beam } \\
5 \mathrm{keV}\end{array}$ & $\begin{array}{c}\text { Beta beam } \\
15 \mathrm{keV}\end{array}$ & $\begin{array}{c}\text { Texono } \\
400 \mathrm{eV}\end{array}$ & $\begin{array}{c}\text { Stopped } \\
\text { pion source }\end{array}$ & Double Chooz & current limit \\
\hline \hline Sensitivity & 450 & 419 & 358 & 406 & 251 & 565 & $860[36]$ \\
\hline
\end{tabular}

TABLE III: Expected sensitivity at $95 \%$ C. L., in $\mathrm{GeV}$, for the mass of a left-right symmetric model extra gauge boson. We consider five different experimental proposals. The current limit is also shown for comparison.

expect its confirmation in this kind of experiments, or their non-observation in the case of other specific models, providing in any case indirect complementary information.

In order to test how the sensitivity to an extra Z signal could change with an upgraded version of these proposals, we show in Fig. 2 the improved sensitivity for each proposal in the case of an increase in mass or time exposure, which reduces the statistical error.

We can see that in the case of extra gauge boson $Z^{\prime}$ the neutrino experimental proposals could only give a complementary information to the current Tevatron constraints [1].

\section{B. Leptoquark models}

A leptoquark is a scalar or vector boson that couples to a lepton and a quark. There are no such interactions in the SM, but they are expected to exist in various extensions of the SM [1], such as the Pati-Salam model [37], grand unification theories based on $S U(5)$ [38, 39] and $S O(10)$ [40] gauge groups and extended technicolor models [41].

The leptoquark contribution effectively (in 4-fermion approximation) can be written as [42]

$$
\begin{aligned}
\varepsilon^{u V} & =\frac{\lambda_{u}^{2}}{m_{l q}^{2}} \frac{\sqrt{2}}{4 G_{F}} \\
\varepsilon^{d V} & =\frac{\lambda_{d}^{2}}{m_{l q}^{2}} \frac{\sqrt{2}}{4 G_{F}},
\end{aligned}
$$

where $\lambda_{u}, \lambda_{d}$ are couplings, $m_{l q}$ is leptoquark mass. This parameterization is given for vector leptoquarks. In the case of scalar leptoquarks, our results should be multiplied by a factor $1 / 2[42]$.

In case of an observation at colliders like LHC and LEP, one can constrain directly the leptoquark mass. The expected sensitivity for LHC could be as high as $1.6 \mathrm{TeV}$ [44]. 


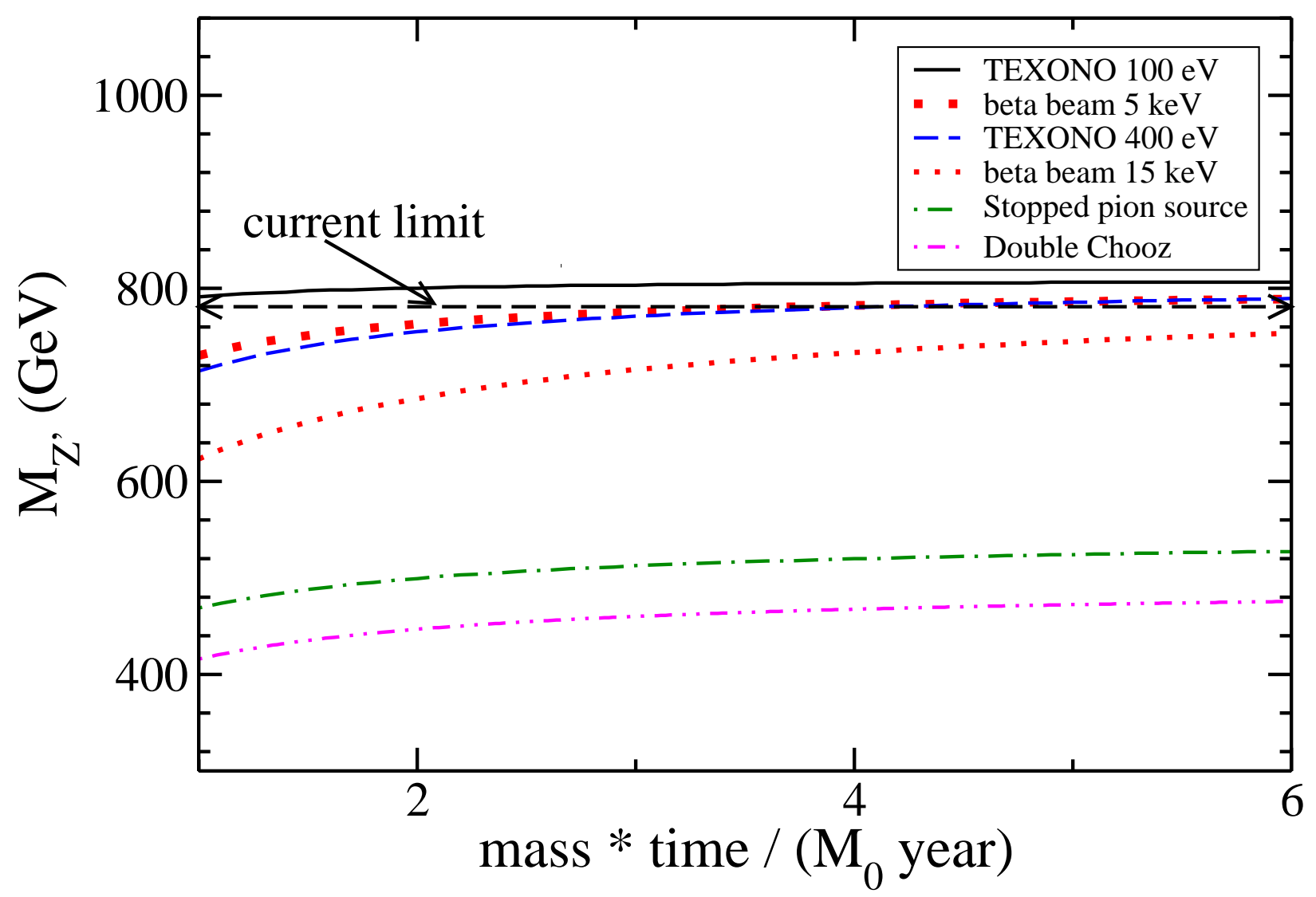

FIG. 2: Sensitivity, at $95 \%$ C. L., to an extra $\chi$ neutral gauge boson coming from $E_{6}$ models for different experimental setups. The dependence on the size of the detector and time of running is shown.

\begin{tabular}{|c|c|c|c|c|c|c|}
\hline Experiment & Texono & Beta beam & Texono & Beta beam & Stopped \\
$100 \mathrm{eV}$ & $5 \mathrm{keV}$ & $400 \mathrm{eV}$ & $15 \mathrm{keV}$ & $\begin{array}{c}\text { current } \\
\text { pion source }\end{array}$ & constraint \\
\hline \hline Sensitivity & 894 & 805 & 805 & 684 & 546 & $298[43]$ \\
\hline
\end{tabular}

TABLE IV: Expected 95 \% C L leptoquark mass sensitivity, in GeV, for future low energy neutrino experiments. The leptoquark effective coupling has been fixed to be $\lambda_{q}^{2} / 4 \pi=1 / 137$.

However, for indirect observations, like our low energy 4-fermion case, one can constrain only the combination $\lambda_{q}^{2} / m_{l q}^{2}$. An extensive list of constraints on the leptoquark couplings and masses is given in Refs. [1, 42]. The current limit for a leptoquark which couples to the first generation of leptons and first generation of quarks is given by

$$
\lambda_{q}^{2} /\left(m_{l q} / 300 \mathrm{GeV}\right)^{2}<0.02
$$




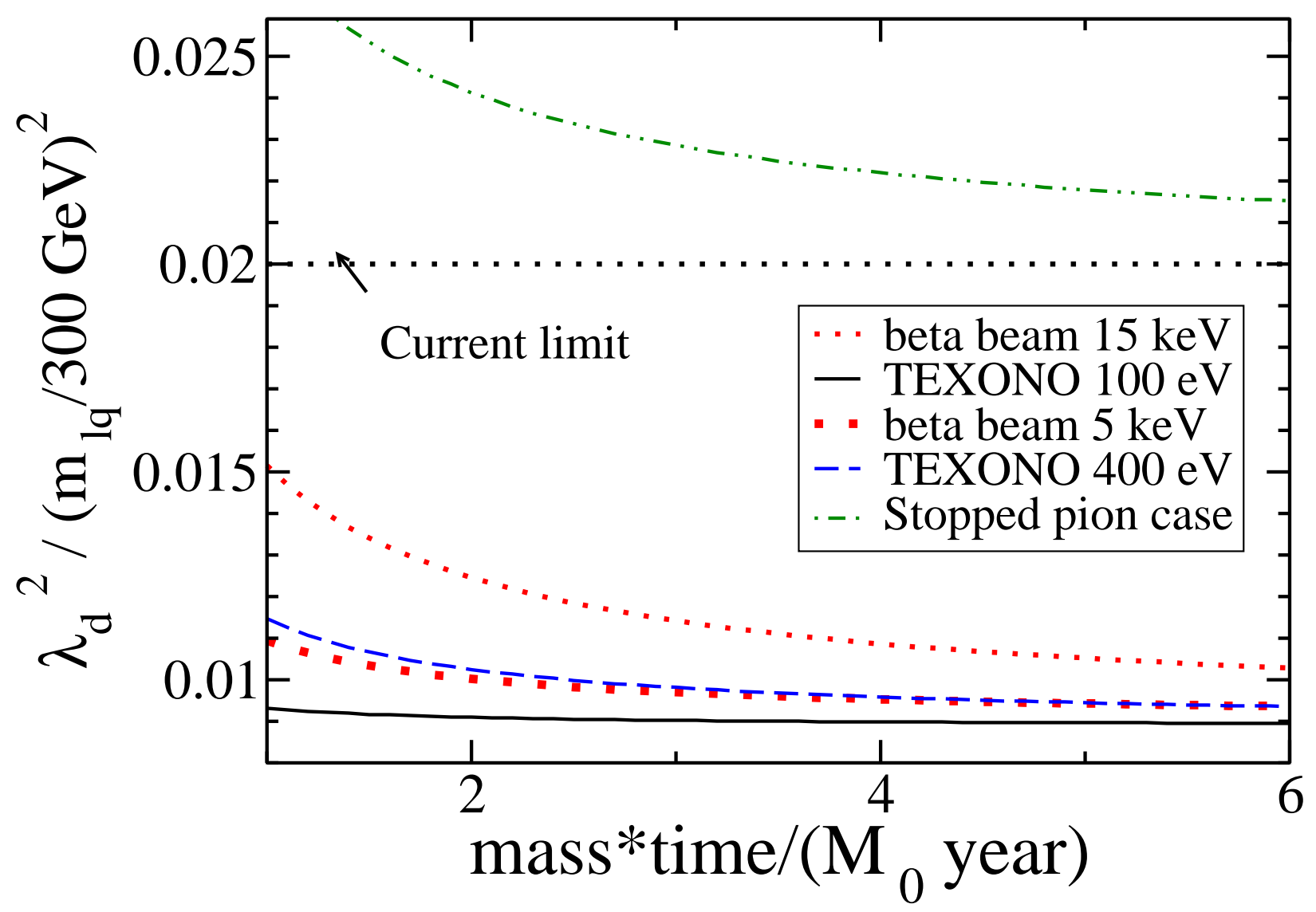

FIG. 3: Sensitivity, at $95 \%$ C. L. to a vector leptoquark coupling for different experimental setups. The limit on the coupling $\lambda_{d}$ will depend on the leptoquark mass $m_{l q}$ that here is chosen to be 300 $\mathrm{GeV}$ in agreement with current literature. The dependence on the size of the detector and time of running is also shown.

We have calculated the sensitivity to the vector first generation leptoquark couplings and masses which is expected at different low energy neutrino experiments already discussed in this work. The results are shown in Fig. 3 where we show the expected sensitivity at $95 \%$ C. L. for each experiment and the possible improvements if the experimental setup could run with a bigger mass or for a longer time.

One can see that the low energy neutrino experiments are very promising for improving the present bounds.

The sensitivities for the case of scalar leptoquark masses for different low energy neutrino experiments are collected in Table IV]. For easy comparison with the bounds given in [1] we have fixed the leptoquark effective coupling at the electroweak value: $\lambda_{q}^{2} / 4 \pi=1 / 137$ and we compute the sensitivity of the scalar leptoquark mass at $95 \%$ C.L. These results also show 


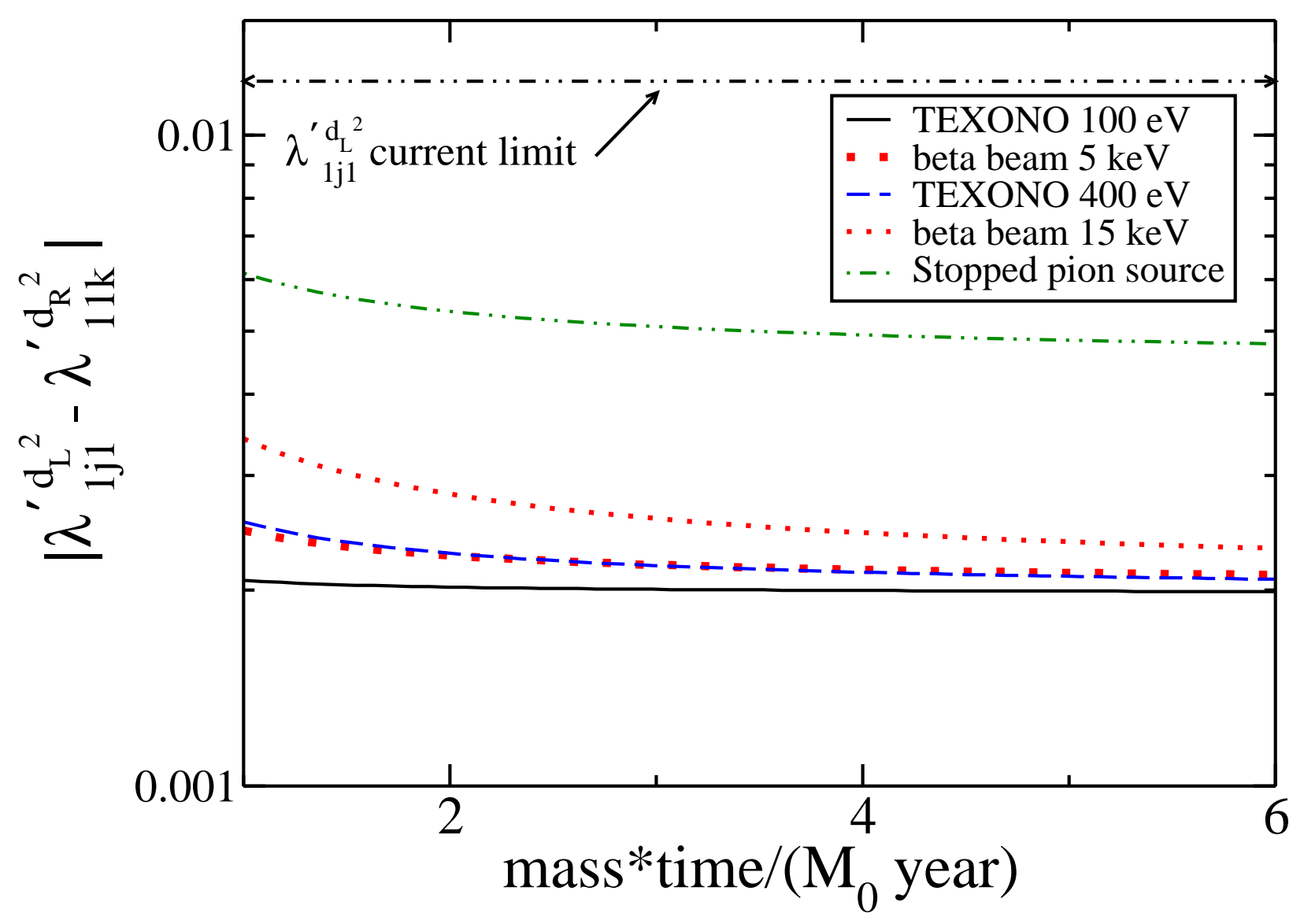

FIG. 4: Sensitivity, at $95 \%$ C. L., to neutral current $R$-parity breaking terms for different experimental setups. The dependence on the size of the detector and time of running is also shown as well as the current limits. See the text for a detailed explanation of these couplings

a big potential for low energy neutrino experiments to give a complementary information about leptoquark masses and couplings.

\section{SUSY with broken R-parity}

In supersymmetric theories, gauge invariance does not imply baryon number (B) and lepton number $(\mathrm{L})$ conservation and, in general, the so called R-parity (defined as $R=$ $(-1)^{3 B+L+2 S}$ where $S$ is the spin) is violated. However, one has to keep the consistency with the non-observation of fast proton decay. One may consider, for instance, the R-parity violating MSSM (imposing baryon number conservation) with a superpotential that contains 
the following $L$ - violating terms [45]:

$$
\begin{gathered}
\lambda_{i j k} L_{L}^{i} L_{L}^{j} \bar{E}_{R}^{k} \\
\lambda_{i j k}^{\prime} L_{L}^{i} Q_{L}^{j} \bar{D}_{R}^{k},
\end{gathered}
$$

where we use the standard notation, $L_{L}, Q_{L}, \bar{E}_{R}$, and $\bar{D}_{R}$ to denote the chiral superfields containing the left-handed lepton and quark doublets and the right-handed charged-lepton and $d$-quark singlets respectively; $i, j, k$ are generation indices. A lepton-Higgs term $(L H)$ can also be included in the superpotential, but it can be rotated away through an appropriate redefinition of the superfields.

At low energies, the heavy Supersymmetry particles can be integrated out and the net effect of the $R$-breaking interactions is to generate effective 4-fermion operators involving the lepton and quark fields.

By considering the case where a single Yukawa coupling (with one flavor structure) is much larger than the others, the effective four-fermion operator generated by $L_{L}^{i} Q_{L}^{j} \bar{D}_{R}^{k}$ takes the same form as in Eq. (8) with the new couplings [45, 46]:

$$
\begin{aligned}
f^{u L} & =\rho_{\nu N}^{N C}\left(\frac{1}{2}-\frac{2}{3} \hat{\kappa}_{\nu N} \hat{s}_{Z}^{2}\right)\left(1-r_{12 k}\left(\tilde{e}_{k R}\right)\right)+\lambda^{u L} \\
f^{d L} & =\rho_{\nu N}^{N C}\left(-\frac{1}{2}+\frac{1}{3} \hat{\kappa}_{\nu N} \hat{s}_{Z}^{2}\right)\left(1-r_{12 k}\left(\tilde{e}_{k R}\right)\right)+\lambda^{d L}-r_{11 k}^{\prime}\left(\tilde{d}_{k R}\right), \\
f^{u R} & =\rho_{\nu N}^{N C}\left(-\frac{2}{3} \hat{\kappa}_{\nu N} \hat{s}_{Z}^{2}\right)\left(1-r_{12 k}\left(\tilde{e}_{k R}\right)\right)+\lambda^{u R} \\
f^{d R} & =\rho_{\nu N}^{N C}\left(\frac{1}{3} \hat{\kappa}_{\nu N} \hat{s}_{Z}^{2}\right)\left(1-r_{12 k}\left(\tilde{e}_{k R}\right)\right)+\lambda^{d R}+r_{1 j 1}^{\prime}\left(\tilde{d}_{j L}\right)
\end{aligned}
$$

where

$$
r_{i j k}(\tilde{l})=\left(\frac{M_{W}^{2}}{g^{2}}\right)\left(\frac{\left|\lambda_{i j k}\right|^{2}}{m_{\tilde{l}}^{2}}\right) .
$$

The factors $\left(1-r_{12 k}\left(\tilde{e}_{k R}\right)\right)$ account for the Fermi coupling constant redefinition $G_{F}=$ $G_{F}^{S M}\left(1+r_{12 k}\left(\tilde{e}_{k R}\right)\right)$ that arise from the modification to the $\mu$ decay due to $R$-breaking interaction. Since the value of the Fermi constant comes from muon decay experiments, we can not get any information on the charged current SUSY parameters and we should concentrate only in the neutral current corrections. As already mentioned in a previous section, a different approach has also been considered, that is the direct detection of $\tau$ leptons in a nearby detector [13].

From Eq. (25) we can see that the $R$ breaking terms appear both in the $f^{d L}$ and $f^{d R}$ 
couplings. We take into account this correlation and we show in Fig. 4 the possible future sensitivity at $95 \%$ C. L. of the neutrino nucleus coherent experiments to the parameter

$$
\lambda_{1 j 1}^{\prime d_{L}^{2}}-\lambda_{11 k}^{\prime d_{R}^{2}}=\frac{\left|\lambda_{1 j 1}^{\prime}\right|^{2}}{\left(\frac{m_{\tilde{d}_{j L}}^{2}}{100 \mathrm{GeV}}\right)}-\frac{\left|\lambda_{11 k}^{\prime}\right|^{2}}{\left(\frac{m_{\tilde{d}_{k R}}^{2}}{100 \mathrm{GeV}}\right)} .
$$

As in previous sections, the possible improvements if the experimental setup could run with a bigger mass or for a larger time is shown in Fig. 4. The current constraints for these parameters are given by $\lambda_{1 j 1}^{\prime d_{L}^{2}} \leq 0.0121$ and $\lambda_{11 k}^{\prime d_{R}^{2}} \leq 0.0001$ [46]. Stringent constraints exist for specific values of $k$ and $j$, for instance, from neutrinoless double beta decay [47] in the particular case $k=j=1\left(\lambda_{111}^{\prime d_{L, R}^{2}} \leq 1.5 \times 10^{-7}\right)$. We can neglect the $\lambda_{11 k}^{\prime d_{R}^{2}}$ parameter and conclude that the perspectives to improve the sensitivity to $\lambda_{1 j 1}^{\prime d_{L}^{2}}$ are quite promising for this type of experiments.

\section{CONCLUSIONS}

We have shown that low energy neutrino experiments could provide independent and complementary information on $Z^{\prime}$, leptoquark masses and couplings and R-parity violating SUSY interactions. We have calculated the potential of various future low energy neutrino experiments to either confirm the discovery of extra heavy gauge bosons at LHC or to constrain their masses.

As concrete coherent neutrino-nuclei interaction proposals, we have discussed the TEXONO case, the stopped pion source with a noble gas detector and the beta beams. In the neutrino-electron-scattering case we have concentrated on the Double Chooz experiment. We have found that a coherent neutrino-nuclei scattering using reactor neutrinos, such as the TEXONO proposal, or a beta-beam neutrino source, could have a high sensitivity to new interactions coming from leptoquarks or $R$-parity breaking SUSY, and we showed that the case of a stopped-pion source experiment could also improve the current $R$-parity breaking SUSY constraints. On the other hand, for this kind of experiments an improved constraint to extra heavy neutral gauge bosons seems to be difficult.

For the particular case of leptoquarks, we have found that all the discussed low energy neutrino experiments have the potential to improve the present bound on leptoquark masses and couplings. In particular, the sensitivity to the vector leptoquark mass is of the order 
of $800 \mathrm{GeV}$, assuming an electroweak value of the coupling, $\lambda_{q}^{2} / 4 \pi=1 / 137$. For the case of supersymmetry with broken R-parity, the perspectives to improve the constraint on the $\lambda_{1 j 1}^{\prime}$ and the corresponding mass for the $\tilde{d}_{L}$ are also very promising for all the experimental setups.

Finally, we would like to remark that low energy neutrino experiments have great potential to provide us an indirect information about high energy physics and therefore strongly complement accelerator experiments.

\section{Acknowledgments}

We kindly thank for very productive discussions to G. Centenario and M. Hirsch. This work has been supported by CONACyT, SNI-Mexico and PAPIIT project No. IN113206. TIR was supported by the Marie Curie Incoming International Fellowship of the European Community and he also acknowledges partial support from the Russian foundation for basic research (RFBR) and from the RAS Program "Solar activity". TIR thanks the Physics Department of CINVESTAV for the hospitality during the visit when part of this work was done.

[1] W. M. Yao et al. [Particle Data Group], J. Phys. G 33 (2006) 1.

[2] H. T. Wong, H. B. Li, J. Li, Q. Yue and Z. Y. Zhou, J. Phys. Conf. Ser. 39 (2006) 266 arXiv:hep-ex/0511001.

[3] K. Scholberg, Phys. Rev. D 73 (2006) 033005 arXiv:hep-ex/0511042.

[4] A. Bueno, M. C. Carmona, J. Lozano and S. Navas, Phys. Rev. D 74 (2006) 033010.

[5] F. Ardellier et al. [Double Chooz Collaboration], arXiv:hep-ex/0606025; F. Ardellier et al., arXiv:hep-ex/0405032.

[8] G. C. McLaughlin and C. Volpe, Phys. Lett. B 591, 229 (2004) arXiv:hep-ph/0312156.

[7] J. Barranco, O. G. Miranda and T. I. Rashba, JHEP 0512 (2005) 021 arXiv:hep-ph/0508299].

[8] G. C. McLaughlin and C. Volpe, Phys. Lett. B 591, 229 (2004) arXiv:hep-ph/0312156].

[9] A. de Gouvea and J. Jenkins, Phys. Rev. D 74, 033004 (2006) arXiv:hep-ph/0603036.

[10] H. T. Wong and H. B. Li, Mod. Phys. Lett. A 20, 1103 (2005). 
[11] M. Moretti, C. Broggini and G. Fiorentini, Phys. Rev. D 57, 4160 (1998).

[12] R. Adhikari, S. K. Agarwalla and A. Raychaudhuri, Phys. Lett. B 642, 111 (2006) arXiv:hep-ph/0608034.

[13] S. K. Agarwalla, S. Rakshit and A. Raychaudhuri, arXiv:hep-ph/0609252.

[14] P. Huber and T. Schwetz, Phys. Rev. D70, 053011 (2004), hep-ph/0407026.

[15] K. Schreckenbach, G. Colvin, W. Gelletly and F. Von Feilitzsch, Phys. Lett. B 160, 325 (1985).

[16] P. Vogel and J. Engel, Phys. Rev. D 39, 3378 (1989).

[17] V. I. Kopeikin, L. A. Mikaelyan and V. V. Sinev, Phys. Atom. Nucl. 60 (1997) 172 [Yad. Fiz. 60 (1997) 230].

[18] P. Zucchelli, Phys. Lett. B 532 (2002) 166.

[19] C. Volpe, J. Phys. G 30 (2004) L1 arXiv:hep-ph/0303222.

[20] J. Serreau and C. Volpe, Phys. Rev. C 70 (2004) 055502 arXiv:hep-ph/0403293.

[21] K.S. Krane, "Introductory Nuclear Physics", Ed. John Wiley and Sons (1998).

[22] I. Feister Phys. Rev. 78 (1950) 375.

[23] O. G. Miranda, V. Semikoz and J. W. F. Valle, Phys. Rev. D 58 (1998) 013007 arXiv:hep-ph/9712215.

[24] J. Barranco, O. G. Miranda, C. A. Moura and J. W. F. Valle, Phys. Rev. D 73, 113001 (2006) arXiv:hep-ph/0512195.

[25] M. Dittmar, A. S. Nicollerat and A. Djouadi, Phys. Lett. B 583 (2004) 111 arXiv:hep-ph/0307020.

[26] T. G. Rizzo, arXiv:hep-ph/0610104.

[27] M. C. Gonzalez-Garcia and J. W. F. Valle, Nucl. Phys. B 345 (1990) 312.

[28] M. Cvetic and B. W. Lynn, Phys. Rev. D 35, (1987) 51.

[29] J. Erler and P. Langacker, Phys. Lett. B 456, 68 (1999) arXiv:hep-ph/9903476.

[30] Rabindra N. Mohapatra Unification and Supersymmetry Springer 2003 and references therein.

[31] J. Engel, Phys. Lett. B 264 (1991) 114.

[32] S. P. Ahlen, F. T. Avignone, R. L. Brodzinski, A. K. Drukier, G. Gelmini and D. N. Spergel, Phys. Lett. B 195, 603 (1987).

[33] K. Freese, J. A. Frieman and A. Gould, Phys. Rev. D 37, 3388 (1988).

[34] L. M. Sehgal and M. Wanninger, Phys. Lett. B 171, 107 (1986).

[35] J. Polak and M. Zralek, Nucl. Phys. B 363, 385 (1991). 
[36] K. m. Cheung, Phys. Lett. B 517, 167 (2001) arXiv:hep-ph/0106251].

[37] J. C. Pati and A. Salam, Phys. Rev. D 10, 275 (1974).

[38] H. Georgi and S. L. Glashow, Phys. Rev. Lett. 32, 438 (1974).

[39] I. Dorsner, P. F. Perez and R. Gonzalez Felipe, Nucl. Phys. B 747, 312 (2006) arXiv:hep-ph/0512068]; I. Dorsner and P. F. Perez, Nucl. Phys. B 723, 53 (2005) arXiv:hep-ph/0504276.

[40] H. Fritzsch and P. Minkowski, Annals Phys. 93, 193 (1975).

[41] E. Farhi and L. Susskind, Phys. Rept. 74, 277 (1981).

[42] S. Davidson, D. C. Bailey and B. A. Campbell, Z. Phys. C 61, 613 (1994) arXiv:hep-ph/9309310.

[43] S. Chekanov et al. [ZEUS Collaboration], Phys. Rev. D 68, 052004 (2003) arXiv:hep-ex/0304008.

[44] A. Belyaev, C. Leroy, R. Mehdiyev and A. Pukhov, JHEP 0509, 005 (2005) arXiv:hep-ph/0502067.

[45] V. D. Barger, G. F. Giudice and T. Han, Phys. Rev. D 40, 2987 (1989).

[46] M. Chemtob, Prog. Part. Nucl. Phys. 54, 71 (2005) arXiv:hep-ph/0406029.

[47] M. Hirsch, H. V. Klapdor-Kleingrothaus and S. G. Kovalenko, Phys. Rev. Lett. 75, 17 (1995). 